\title{
Thermally mediated control of liquid microdroplets at a bifurcation
}

\author{
Yit-Fatt Yap $\uparrow$, Say-Hwa Tan $\dagger+$, Nam-Trung Nguyen $\uparrow \S$, S. M. \\ Sohel Murshed $\uparrow$, Teck-Neng Wong $\dagger$, Levent Yobas $\ddagger$ \\ † School of Mechanical and Aerospace Engineering, Nanyang Technological \\ University, 50 Nanyang Avenue, Singapore 639798 \\ ‡ Institute of Microelectronics, Science Park II, Singapore 1176851
}

\begin{abstract}
.
The ability to precisely control the motion of droplets is essential in droplet-based microfluidics. It serves as the basis for various droplet-based devices. This paper presents a thermal control technique for microdroplets at a bifurcation. Control was achieved using an integrated microheater that simultaneously induces a reduction in fluidic resistance and thermocapillarity. The temperature of the heater was monitored by an integrated temperature sensor. At a bifurcation with symmetric branches, a droplet can be split into two daughter droplets of controllable sizes or entirely switched into a desired branch. The physics of this phenomenon was investigated with the help of a numerical model. Splitting and switching were demonstrated within an operational temperature range of 25 to $38^{\circ} \mathrm{C}$. The relatively low operational temperature range allows this technique to be used for droplets containing biological samples. The present control concept is not limited to bifurcations, but can be employed in other geometries.
\end{abstract}

$\S$ To whom correspondence should be addressed (mntnguyen@ntu.edu.sg) 


\section{Introduction}

Recently, extensive works have been conducted in the emerging field of microfluidics. As microfluidics is still in the early stage of its development [1], much of its potential has yet to be tapped. Manipulation of fluids can be realized in microdevices with low consumption of samples and reagents. Microfluidic technologies allow chemical and biochemical analysis to achieve high resolution and sensitivity with a shorter analysis time and potentially a lower operation cost. In particular, microdroplets can be employed either in serial or parallel as isolated compartments for different reactions [2]. Droplet-based microfluidic techniques are essential for large scale integration of devices for biological or chemical analysis. Microdroplets provide both physical and chemical isolation for their contents. Droplet-based microfluidic devices have been employed to investigate enzyme kinetics [3], to perform DNA analysis [4] as well as protein crystallization [5]. The study of a single cell by encapsulating them within a droplet has been demonstrated [6]. The synthesis of organic molecules [7], monodispersed nanoparticles [8] and microcapsules of various contents [9] have been performed on these droplet-based platforms. Though impressive, these applications require precise and effective means to manipulate the droplets in a controlled manner. These manipulations include but not limited to droplet formation, merging, breakup and switching.

Passive geometrical control of microdroplets was previously reported. To break a droplet into two daughter droplets in a T-junction, Link et al. [10] used the lengths of the branches to control the size ratio of the two daughter droplets. The idea is based on the fact that the difference in the length of the branches leads to a difference in their fluidic resistances. The larger daughter droplet forms in the shorter branch with a lower fluidic resistance.

For active control, different forces can be employed to manipulate microdroplets in microchannels. These forces can be classified as either body forces or interfacial forces. Generally, a body force is proportional to the characteristic volume or to the cube of a characteristic length. As the size of the system shrinks, body forces decay rapidly. This seems to suggest that they are not favorable in micro-scale systems. However, certain body forces increase with the potential gradient that creates them. These include, in a continuum sense, magnetic and electric forces. Such behaviour renders these volumetric forces to be actually proportional to the square of the characteristic length. As a result, a magnitude comparable to that of interfacial forces can be achieved. Since microdroplets have to be responsive to the field that creates the body forces, special fluids such as ferrofluids are required. Ferrofluids are suspensions of ferromagnetic nano-particles in suitable carrier liquids [11]. With the inherited magnetic properties, magnetic control of ferrrofluid droplets can be achieved [12]. Previously, our group has demonstrated that magnetic force on a ferrofluid plug is impressively strong to be used for actuation for miniaturized polymerase chain reaction (PCR) devices [13]. Link et al. [14] reported a method of electric control of droplets in a continuous-flow platform where charged droplets were manipulated by electric fields induced by electrodes. Manipulation 
functions such as formation, merging, breakup and sorting were achieved.

Miniaturization leads to a large surface to volume ratio and dominant interfacial forces. Effective manipulation in microscale can therefore be achieved utilizing interfacial forces. One of the well known interfacial forces is the interfacial tension force. The interfacial tension force consists of two components: i.e. the capillary force induced by curvature and the Marangoni force induced by interfacial tension gradient. The Marangoni force drives a droplet in the direction of decreasing interfacial tension. A gradient in interfacial tension can be created by a gradient of either surfactant concentration, electric potential or temperature [15]. The associated phenomena are termed solutocapillarity, electrocapillarity and thermocapillarity, respectively. Maintaining a surfactant concentration gradient in solutocapillarity is not trivial. One way to achieve is is to have surfactant species generated at one electrode and consumed at another. The concept of maintaining surfactant concentration gradient was used to manipulate the magnitude and direction of spatial gradients in interfacial tension and to guide droplets of organic liquids through simple fluidic networks [16]. Besides, the use of different spatial light intensity to activate photo-sensitive surfactant to maintain the required concentration gradient was also investigated [17]. Electrocapillarity was used to drive a liquid mercury droplet in an electrolyte-filled microchannel [18]. Investigation on thermocapillarity of a droplet in an unbounded domain [19], near a solid wall [20], within a capillary [21] and parallel plates [22] provides important insights to the possibility of its application in droplet-based microfluidics. Baroud et al. [23, 24] used a focused laser beam to manipulate microdroplets. In their experiments, the interfacial tension increases with increasing temperature. Thus, the locally induced heat causes thermocapillary force that block the droplets. Further use of thermocapillarity for droplet manipulation remains to be explored.

The passive breakup process of microdroplets and microbubbles were investigated both experimentally and numerically in the past. Garstecki et al. [25, 26] studied the dependence of the breakup process on the flow rates. The phenomenon of blocking and pinching was identified as one of the key mechanisms for breakup of droplets and bubbles at a T-junction [26, 27]. Recently, Dollet et al. [28] investigated experimentally the role of the channel geometry on the breakup process. De Menech [29] used the phase-field method to model the passive breakup process of a droplet at a bifurcation. The dependence of this process on the flow rates, surface tension and viscosities were numerically investigated. The passive transport of microdroplets and microbubbles in microchannels were also reported in the past. Fuerstman et al. [30] investigated the pressure drop across a bubble train in a straight microchannel. According to this study, the pressure drop depends mainly on the number of bubbles in the channel. The relation between the number of droplets/bubbles and the pressure allow the realization of interesting microfluidic logic components [31, 32]. Bypass structures for equalizing pressure drops can be used for passive regulation of droplet trains [33, 34, 35].

This paper presents a thermal control technique for microdroplet at a bifurcation. Preliminary results with a laser micromachined microchannel network and external 


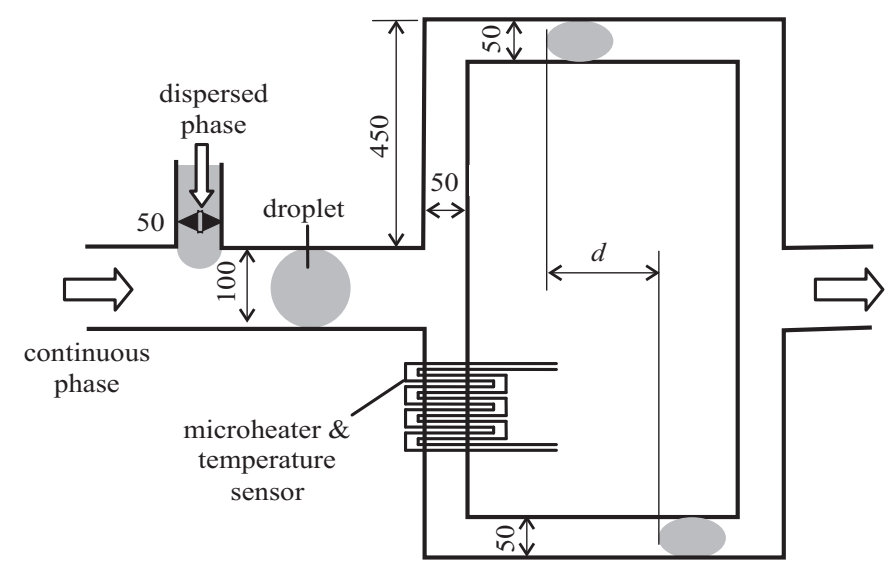

Figure 1. Schematic of the microchannel system (dimensions in $\mu \mathrm{m}$ ).

heating wires were reported earlier by the authors [36]. The control concept is based on the temperature dependent fluidic resistance of the branches and the thermocapillary effect. Both can now be actively adjusted through the temperature field using an integrated microheater and an temperature sensor.

\section{Materials and Methods}

\subsection{Experimental Investigation}

Figure 1 depicts the schematic concept of the microfluidic device used in our experiments. The device is based on the bifurcation design proposed by Jousse et al. [34]. The channel first splits into two identical arms and subsequently recombines. Due to its symmetry, the resistance of each arm only depends on the number of droplets in it [35]. The device was fabricated using micromachining of glass and polydimethylsiloxane (PDMS). To begin with, the thin-film platinum microheater and the temperature sensor were patterned using photolithography and lift-off technique. The adhesion of platinum to the glass wafer was improved by the deposition of a thin titanium layer. The glass wafer with the patterned microheater and the temperature sensor was then coated with a thin layer of PDMS to serve as the bottom wall of the microchannel network.

The microchannel network of a $30-\mu \mathrm{m}$ depth with the dimensions shown in Fig. 1 was fabricated on PDMS using soft lithography. The master mould was photolithographically fabricated from the thick-firm resist SU-8 with a low-cost mask. This moulded PDMS layer was subsequently bonded onto the PDMS-coated glass wafer after an oxygen plasma treatment of both surfaces. The bonded device was then tempered at $150^{\circ} \mathrm{C}$ for 3 hours to ensure an adequate bonding. The temperature sensor was calibrated so that its electrical resistance can be used for in-situ temperature measurement.

The experiments were conducted using de-ionized water with $0.05 \% \mathrm{w} / \mathrm{w}$ fluorescence dye (Sigma F6377) as the dispersed phase and mineral oil (Sigma M5904) 
with $2 \% \mathrm{w} / \mathrm{w}$ surfactant Span 80 (Sigma S6760) as the continuous phase. The flow rates of the dispersed and continuous phases were set to $Q_{\mathrm{d}}=0.5 \mu \mathrm{l} / \mathrm{h}$ and $Q_{\mathrm{d}}=10 \mu \mathrm{l} / \mathrm{h}$, respectively. The large flow rate ratio of 20 makes sure that only one droplet exists in the bifurcation loop. This experimental condition eliminates the influence of droplet number in each branch. These flow rates were maintained constant throughout all experiments.

Observation of the droplet evolution was made using an epi-fluorescent inverted microscope (model ECLISPE TSE2000-S) with a filter (Nikon B-2A, excitation filter for 450-490nm, diachronic mirror for $505 \mathrm{~nm}$, and emission filter for $520 \mathrm{~nm}$ ) using both fluorescent and phase-contrast modes. For the purpose of analysis, the droplet images were recorded by a sensitive interline transfer charge coupled device (CCD) camera (HiSense MKII) with a spatial resolution of $1344 \times 1024$ pixels. The recorded images were processed by a customized MATLAB program to measure the droplet diameters and its positions.

\subsection{Numerical Investigation}

The motion of both the continuous and dispersed phases is governed by the incompressible Navier-Stokes and the energy equations. These equations are strongly coupled together. A level-set method [37] coupled with a global mass correction scheme [38] is employed to capture the interface between the two phases. Interfacial tension is treated within the framework of the Continuum Surface Force model [39]. With the heater turned on, a non-symmetric temperature field is generated. This gives rise to variations in the thermo-physical properties of the liquid phases, in particular interfacial tension and viscosities. The interfacial tension and the viscosities of both phases were measured and empirically fitted against temperature [40]:

$$
\begin{aligned}
\frac{\sigma}{\sigma_{0}} & =\exp (-0.0144 \Delta T) \\
\frac{\mu_{\mathrm{c}}}{\mu_{\mathrm{c} 0}} & =\exp (-0.0344 \Delta T) \\
\frac{\mu_{\mathrm{d}}}{\mu_{\mathrm{d} 0}} & =-9 \times 10^{-6} \Delta T^{3}+6 \times 10^{-4} \Delta T^{2}-2.55 \times 10^{-2} \Delta T+1
\end{aligned}
$$

where $\Delta T=T-T_{0}$ is the difference to the reference temperature of $T_{0}=25^{\circ} \mathrm{C}$. The interfacial tension, the viscosity of the carrier fluid and the viscosity of the droplet fluid at the reference temperature are $\sigma_{0}=3.65 \times 10^{-3} \mathrm{~N} / \mathrm{m}, \mu_{\mathrm{c}, 0}=2.38 \times 10^{-2}$ Pas, $\mu_{\mathrm{d}, 0}=9 \times 10^{-4}$ Pas, respectively.

At the inlet, the velocity profile is assumed to be fully developed. Outflow condition is enforced at the outlet. No slip and no penetration conditions are applied at the walls. As for the energy equation, a conjugate heat transfer problem is considered with temperature at the edge of the PDMS specified at the ambient temperature of $25^{\circ} \mathrm{C}$. Within the microchannel, the temperature at the location where the microheater is located is set to the temperature measured in the experiments. The governing equations are solved using a finite volume method [41]. 

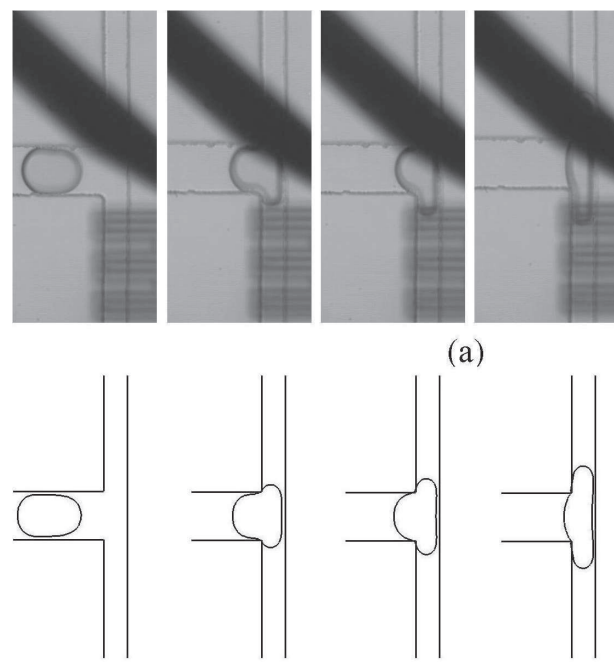

(b)

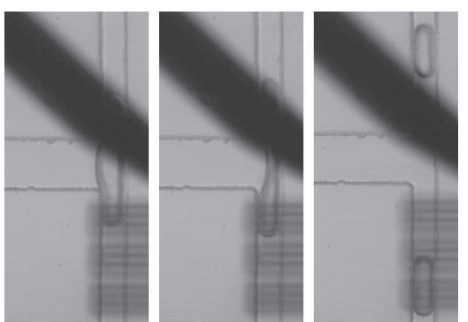

(a)

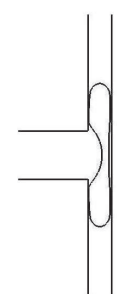

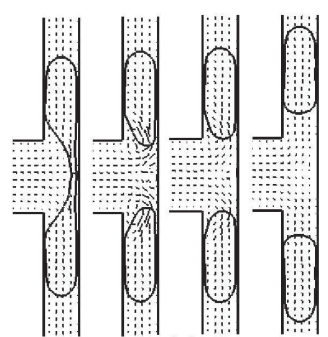

(c)

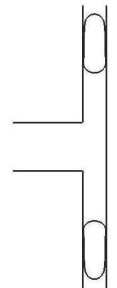

(d)

Figure 2. Passive splitting of a droplet into two daughter droplets of equal size at $T=25 \circ$ C: (a) Experimental snapshots; (b) Simulation results; (c) Formation and decay of vortices and (d) Capillary forces acting on the daughter droplets.

\section{Results and Discussions}

\subsection{Passive Splitting of Droplets}

Figure 2 shows the evolution of the droplet in the bifurcation when the heater was switched off. The temperature was maintained at $25 \circ \mathrm{C}$. The droplet splits into two daughter droplets of identical sizes because of the symmetric nature of the flow field. The snapshots of the splitting process obtained experimentally and numerically are given in Figs. 2(a) and (b) respectively. Although the numerical model is only twodimensional and therefore is of a qualitative nature, it does capture the deformation of the droplet entering the bifurcation reasonably well and reveal the essential features of the splitting process.

Upon arriving at the bifurcation, the droplet blocks the junction and consequently creates a build-up of upstream pressure. This phenomenon was observed experimentally during a droplet formation process by Guillot et al. [27] and Garstecki et al. [27, 26]. The pressure build-up squeezes the droplet into the shape of a dumbbell. The two forming daughter droplets at the two ends of the original droplet are joined by a thin thread. These two forming daughter droplets further block the flow of the continuous phase in the branches accelerating the build-up of the upstream pressure. The forming daughter droplets are pushed further into the branches and results in a thinning of the thread. The droplet thread finally breaks. The two daughter droplets detach from each other and move into their respective branches. The same results were achieved with the phase-field method by De Menech [29].

Figure 2(c) shows the velocity field before and after the splitting of the droplet. The velocity field is highly complicated with vortices formed adjacent of the daughter 
droplets. For the ease of explanation, a schematic of the two daughter droplets just after the thread breaks is depicted in Figure 2(d). Upon detachment, a pointed tail forms at the rear of each daughter droplet from the broken droplet thread. Such a sudden creation of pointed interfaces with large curvature instantly generates strong capillary forces. Given the symmetry of the flow field, only the effect of the capillary forces on the lower formed daughter droplet is discussed. In Fig. 2(d), $\tau_{1}$ is the capillary force acting on lower daughter droplet at the pointed tail. The strength and direction of the capillary force is clearly manifested in the velocity field at the pointed tail. This force pulls the pointed tail towards the center of the lower daughter droplet, effectively shortening the tail in the process. The shear created by $\tau_{1}$ is so large that the tail is able to drag along the fluid particles of the continuous phase adjacent to it and dramatically increases the continuous phase velocity. This can be seen from the sudden increase in the continuous phase velocity around the detached daughter droplets. However, the lower daughter droplet is still blocking the lower branch. The blocking effect drives the fluid particles of the continuous phase to circulate at the junction. As the tail of the droplet is shortened, it becomes more rounded, thus possesses a smaller curvature. The decrease in curvature is accompanied by decreasing capillary force. As result, the circulation decays as the capillary force at the tail is no longer strong enough to sustain it. Similar phenomenon occurs to the upper daughter droplet resulting in the formation and decay of the upper circulation.

The daughter droplets attain their steady-state shape as they travel downstream under the influence of interfacial tension. The two daughter droplets are of equal size. From the above observation, a droplet of size smaller than the width of the branch is not likely to split because it fails to create the required pressure build-up for splitting. It can only flow into one of the branches depends on the perturbation of the flow conditions in these branches.

\subsection{Thermally Mediated Splitting of Droplets}

Figure 3(a) shows the snapshots of thermally mediated control of droplet splitting at the bifurcation. The heater was switched on, and the measured temperature at the heater is $36 \circ \mathrm{C}$. The heater creates an asymmetric temperature field with strong temperature gradient around the heater in the lower branch as shown in Fig. 3(b). As heat is mainly carried downstream, obvious temperature gradient is not expected in the upper branch. This results in a variation in viscosity due to its temperature dependent nature and a variation in interfacial tension giving rise to thermocapillarity. With these two driving factors, the droplet still splits, but a larger daughter droplet was formed in the lower branch, Fig. 3(a). This larger daughter droplet flows faster than the smaller daughter droplet in the upper branch.

Simulation results were invoked to gain further insights into the above observation. The splitting process is shown in Fig. 3(c). The fluid viscosity in the lower heated branch is smaller. The fluid experiences a smaller fluidic resistance. As the two branches are 

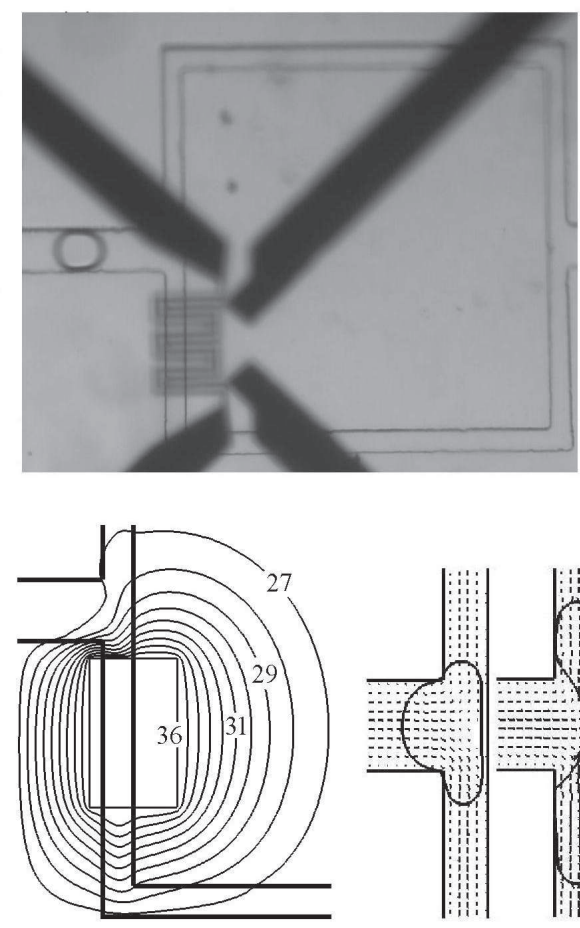

(b)

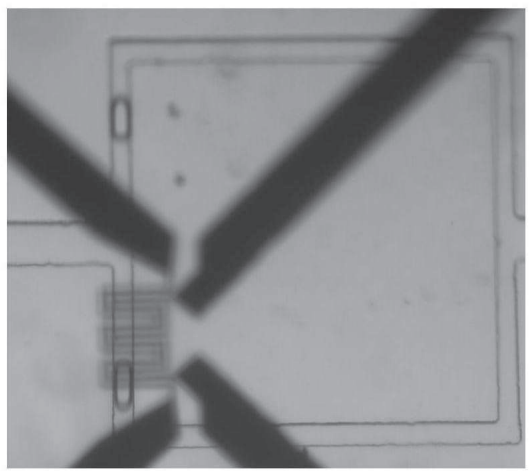

(a)
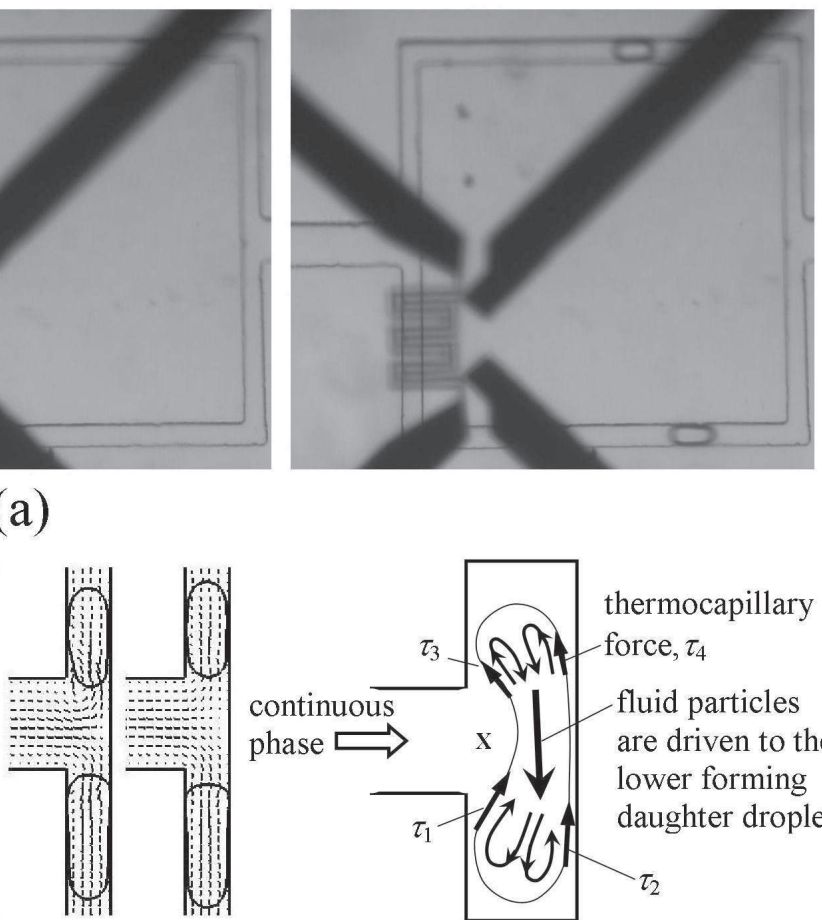

(c)

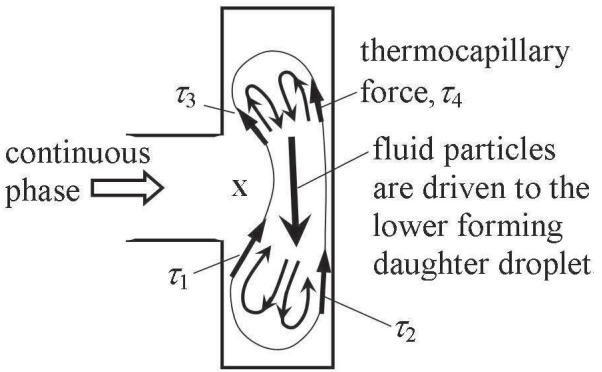

(d)

Figure 3. Thermal control of splitting a droplet into two daughter droplets of controllable sizes at T=36 C: (a) Snapshots of the experiment; (b) Temperature field in the microchannel network and PDMS; (c) Unequal splitting of a droplet in a Tjunction with temperature field.; (d) Thermocapillary forces acting on the droplet in a non-uniform temperature field.

maintained at the same pressure difference, the lower branch has a larger volumetric flow rate. The fluid in the lower branch flows at a faster speed as the cross sectional areas of the both branches are identical. When the droplet enters the junction, a larger portion of the droplet is already drawn into the lower branch. The role of the thermocapillary force is now explained. Figure 3(d) shows the thermocapillary forces $\tau_{1}, \tau_{2}, \tau_{3}$ and $\tau_{4}$ acting at different locations of the droplet interface. These forces, upwardly directed, induce a secondary flow within the droplet of the following nature. The thermocapillary forces drive the droplet fluid particles adjacent to the interface upwards into the upper forming daughter droplet. This effect depletes the droplet thread and accelerates its thinning. The accumulation of the droplet fluid particles in the upper forming daughter droplet tends to inflate it. It should be noted that the magnitude of the interfacial tension in upper branch is larger than that of the lower branch given the lower temperature in the upper branch. Creating larger interfacial area at the tip of the formed upper droplet to accommodate additional droplet fluid particles is not favorable as more energy is required. The droplet fluid particles then turn and flow downward into the lower forming daughter droplet via the center of the droplet thread. When these particles reach the tip of the lower formed daughter droplet, a larger interface can be favorably created as the interfacial tension is smaller and thus less energy is required. As a result, more droplet 


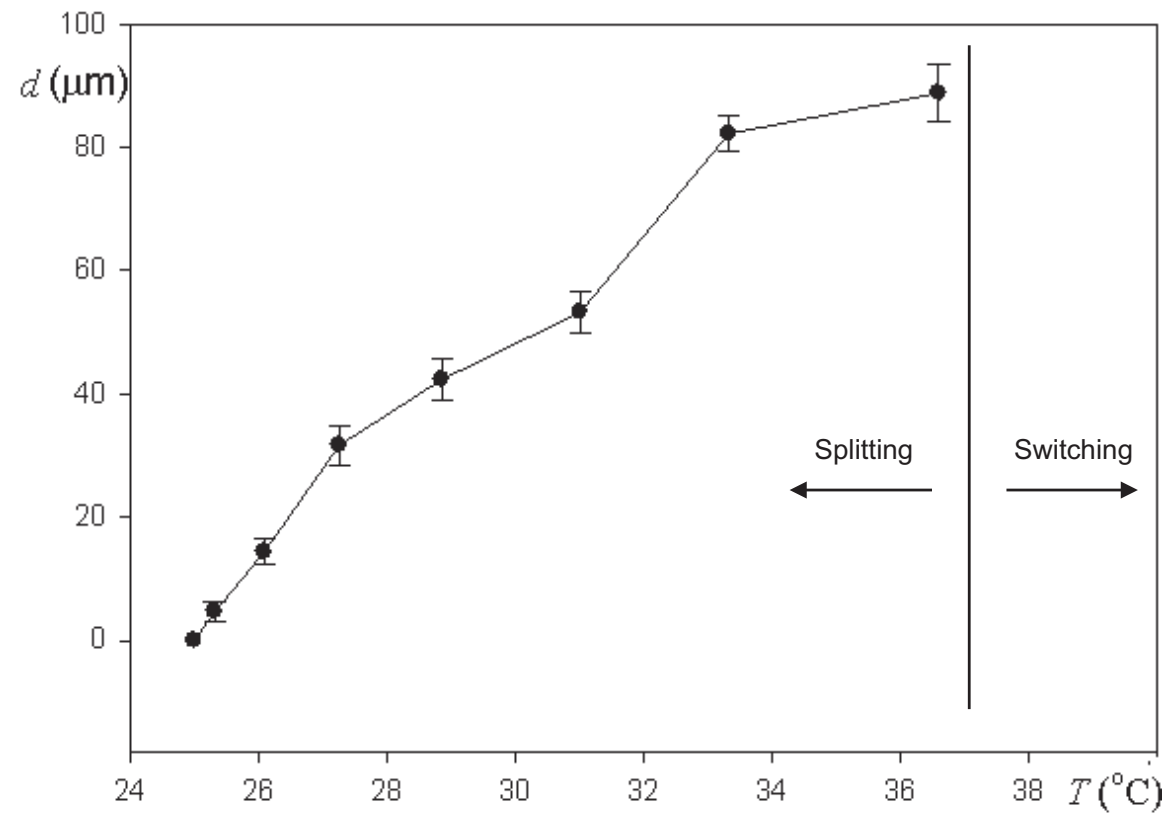

Figure 4. Variation of the distance between the two daughter droplets with temperature.

fluid particles accumulate in the formed lower droplet. With the coupling of these two driving factors, upon the detachment of the two daughter droplets, the lower daughter droplet is larger.

The mechanism in generating flow recirculation is similar to the case where the heater is turned off. However, since now the capillary forces at the pointed tails of the detached daughter droplets are no longer of the same magnitude, the strength of the induced circulation is different. The upper daughter droplet experiences a larger capillary force. Thus, the upper circulation is stronger and decays slower.

The difference in the flow velocity in the two branches can be measured indirectly from the experiments. The separation distance between the two daughter droplets (Fig.1) indicates the relative velocity between them. This distance shown in Fig. 4 is measured for temperature up to $T=36^{\circ} \mathrm{C}$. When the heater is turned off, the two daughter droplets flow with the same speed, giving a zero separation distance. Upon switching on the heater, the separation distance increases with increasing temperature and consequently decreasing viscosity. The two droplets flow at increasing different speeds due to a much lower viscosity and thus fluidic resistance in the lower branch.

Figure 5 shows the size ratio of the upper droplet to the lower one. A size ratio of around 0.85 is achieved with a temperature of $T=36^{\circ} \mathrm{C}$. To achieve a pressure build-up at position marked with "X" in Fig. 3(c) and the subsequent thinning and breaking of the thread joining the two formed daughter droplets, the daughter droplets have to block their respective branch. The smallest size of the formed daughter droplet sufficient to block the branch depends on the size of the branch itself. A smaller branch would require a smaller formed daughter droplet. This requirement sets a lower limit on the 


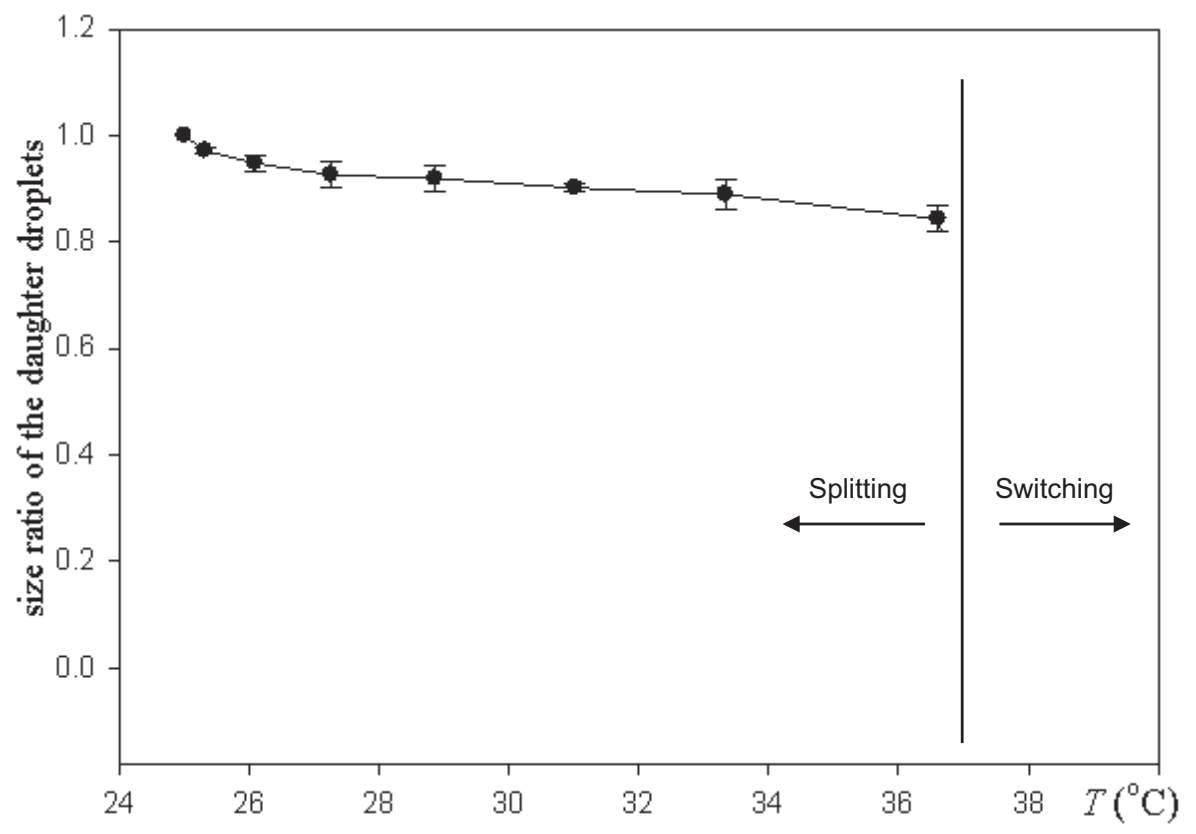

Figure 5. The ratio of the size of the larger daughter droplet to the smaller daughter droplet at different temperature.

size of the daughter droplets that can be formed for a given branch size. We could not form a daughter droplet smaller than the branch width in the above mentioned manner. Beyond the temperature of $T=36^{\circ} \mathrm{C}$, the whole droplet is switched into the lower branch as described next.

\subsection{Thermal Mediated Switching of Droplets}

At $T=40^{\circ} \mathrm{C}$, the combined reduction of flow resistance and thermocapillarity is so strong that the droplet no longer splits but switches entirely into the lower branch, Fig. 6(a). Figure 6(b) shows the simulated flow field around the droplet. The asymmetrical shape of the droplet is obvious with a smaller upper forming droplet which blocks the upper branch. The pressure build-up pushes the two forming daughter droplets into the respective branch. This is accompanied by a thinning of the droplet thread. However, given the different size in the formed daughter droplet, the ability to withstand the pressure build-up is different. The smaller upper droplet fails to withstand further pressure build-up eventually and the pressure forces the creation of a small gap between the upper droplet and the wall. The sudden release of pressure accelerates the continuous phase into the upper branch via the gap. This generates a large shear stress, upwardly directed, on the interface of the upper formed daughter droplet, creating two recirculations within it. These recirculations accelerate the fluid particles in the upper droplet downwards into the lower droplet. The upper droplet becomes smaller. The droplet thread does not break because of two reasons. First, there is no further pressure build up thinning it. Second, the flow of the droplet fluid particles from the upper 

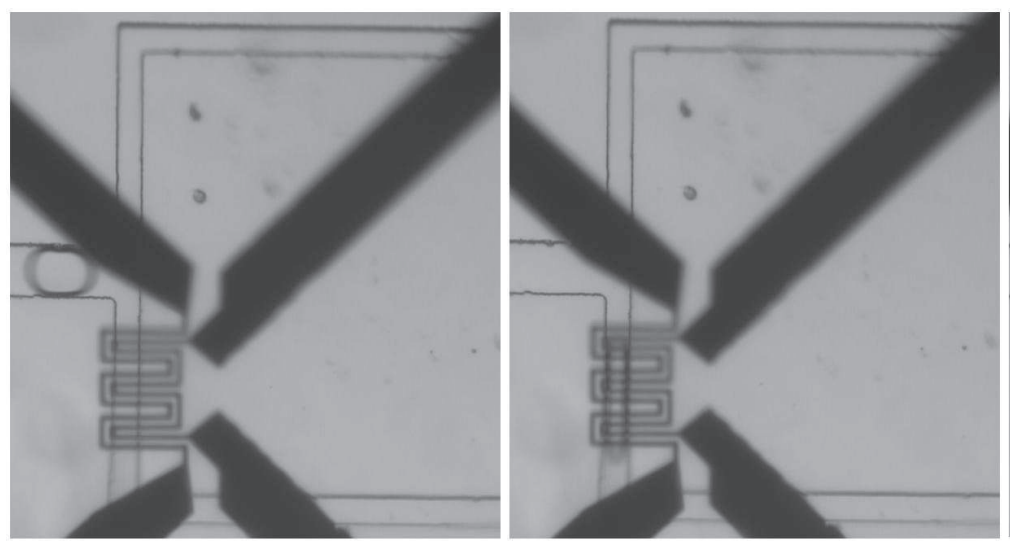

(a)
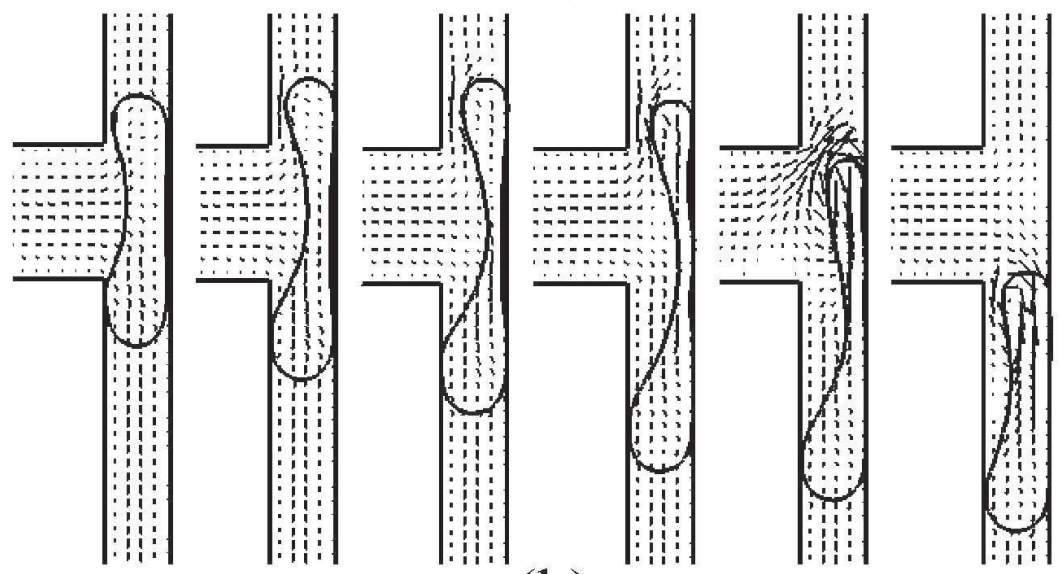

(b)

Figure 6. Thermally mediated switching a droplet into the lower heated branch at $T=40^{\circ} \mathrm{C}$ : (a) Snapshots of experiment; (b) Thermal switching of a droplet in a T-junction; (c) Circulation in the upper formed daughter droplet.

daughter droplet to the lower daughter droplet replenishes the thread. As a result, the upper droplet does not detach but merges with the lower droplet. In the process, the whole droplet is switched into the lower branch.

The relative importance of resistance reduction and thermocapillarity in the experiments is now assessed. Figure 7 shows the variation of the normalized viscosity of the continuous phase and the normalized interfacial tension as temperature difference increases [40]. Normalization is performed with the nominal values at $T=25^{\circ} \mathrm{C}$. For a temperature difference of $T=20^{\circ} \mathrm{C}$, it can be seen that the viscosity decreases more than $50 \%$. However, the interfacial tension decreases only around $30 \%$. Therefore, the variation of viscosity plays a more important role in controlling the droplet.

\section{Conclusions}

The present study investigates thermally mediated control of droplets at a bifurcation. This type of control was achieved by generating a temperature field to induce 


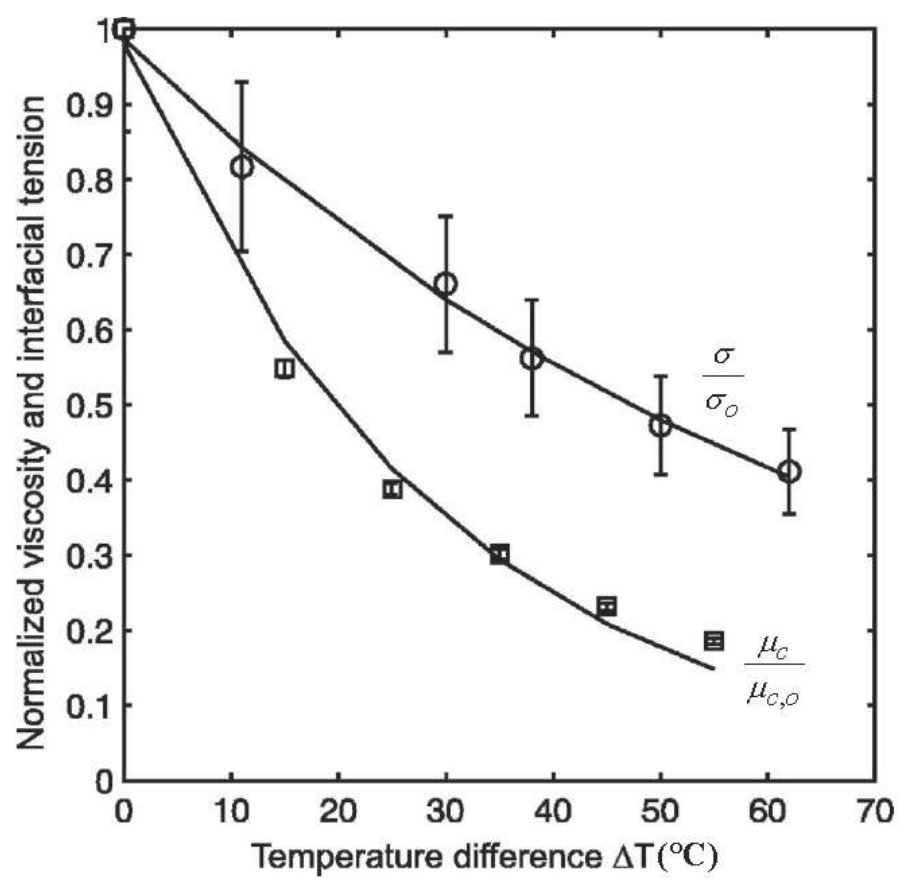

Figure 7. Variation of normalized viscosity of the continuous phase and normalized interfacial tension with temperature difference [40].

thermocapillarity and to reduce the fluidic resistance in one of the branches. The combined effect of thermocapillarity and reduction in fluidic resistance increases the tendency for the droplet flowing into the branch with the higher temperature, whereby the motion of the droplet can now be controlled via the generated temperature field. Splitting of the droplet into two daughter droplets of controllable size and switching of the droplet into a desired branch were achieved. Detailed hydrodynamics leading to the control was investigated with the help of a numerical model. The new insights are useful in the design of thermal control for other geometries.

\section{Acknowledgement}

The authors would like to thank the Agency of Science, Technology and Research, Singapore A*Star, SERC Grant No. 0521010108, "Droplet-based micro/nanofluidics" for its financial support.

\section{References}

[1] Whitesides GM 2006 The origins and the future of microfluidics Nature $422368-373$

[2] Song H, Chen DL and Ismagilov RF 2006 Reactions in droplets in microfluidic channels Angew. Chem. Int. Ed. 45 7336-7356

[3] Song H and Ismagilov RF 2003 Millisecond kinetics on a microfluidic chip usingnanoliters of reagents J. of the American Chemical Society $12514613-14619$

[4] Burns MA, Johnson BN, Brahmasandra SN, Handique K, Webster JR, Krishnan M, Sammarco 
TS, Man PM, Jones D, Heldsinger D, Mastrangelo CH, Burke DT 1998 An integrated nanoliter DNA analysis device Science 282 484-487

[5] Zheng B and Ismagilov RF 2005 A microfluidic approach for screening submicroliter volumes against multiple reagents by using preformed arrays of nanoliter plugs in a three-phase liquid/liquid/gas flow Angew. Chem. Int. Ed. 44 2520-2523

[6] He M, Edgar JS, Jeffries GDM, Lorenz RM, Shelby JP and Chiu DT 2005 Selective encapsulation of single cells and subcellular organelles into picoliter- and femtoliter-volume droplets Analytical Chemistry 77 1539-1544

[7] Onal Y, Lucas M and Claus P 2005 Application of a capillary microreactor for selective hydrogenation of $\alpha, \beta$-unsaturated aldehydes in aqueous multiphase catalysis Chemical Engineering and Technology $\mathbf{2 8} 972-978$

[8] Khan SA, Gunther A, Schmidt MA, Jensen MA 2004 Microfluidic synthesis of colloidal silica Langmuir 20 8604-8611

[9] Darhuber AA, Davis JM, Troian SM, Reisner WW 2003 Thermocapillary actuation of liquid flow on chemically patterned surfaces Physics of Fluids 15 1295-1304

[10] Link DR, Anna SL, Weitz DA, and Stone HA 2004 Geometrically mediated breakup of drops in microfluidic devices Physical Review Letters 92 545031-545034

[11] Rosensweig RE 1985 Ferrohydrodynamics Cambridge University Press, Cambridge

[12] Nguyen NT, Beyzavi A, Ng KM, Huang XY 2007 Kinematics and deformation of ferrofluid droplets under magnetic actuation Microfluidics and Nanofluidics 3 571-579

[13] Sun Y, Kwok YC, Nguyen NT 2007 A circular ferrofluid driven microchip for rapid polymerase chain reaction Lab on a Chip 7 1012-1017

[14] Link DR, Grasland-Mongrain E, Duri A, Sarrazin F, Cheng Z, Cristobal G, Marquez M and Weitz DA 2006 Electric control of droplets in microfluidic devices Angew. Chem. Int. Ed. 45 $2556-2560$

[15] Squires TM 2005 Microfluidics: fluid physics at the nanoliter scale Reviews of Modern Physics $\mathbf{9 7 7} 977-1026$

[16] Gallardo BS, Gupta VK, Eagerton FD, Jong LI, Craig VS, Shah RR, Abbott NL 1999 Electrochemical principles of active control of liquids on submillimeter scales Science $\mathbf{2 8 3}$ $57-60$

[17] Shin JJ and Abbott NL 1999 Using light to control dynamic surface tensions of aqueous solutions of water soluble surfactants Langmuir 15 4404-4410

[18] Lee J and Kim CJ 2000 Surface-tension-driven microactuation based on continuous electrowetting Journal of Microelectromechanical System 9 171-180

[19] Young NO, Goldstein JS and Block MJ 1959 The motion of bubbles in a vertical temperature gradient Journal of Fluid Mechanics 6 350-356

[20] Savino R, Monti R and Alterio G 2001 Drops pushing by Marangoni forces Physics of Fluids $131513-1516$

[21] Nguyen NT, Huang XY 2005 Drops pushing by Marangoni forces Japanese Journal of Applied Physics, Part 1: Regular Papers and Short Notes and Review Papers 44 1139-1142

[22] Zhao ZJ, Huang XY, Nguyen NT and Abgrall P 2008 Thermocapillary actuation of droplet in a planar microchannel Microfluidics and Nanofluidics 5 205-214

[23] Baroud CN, Delville JP, Gallaire F, Wunenburger R 2007 Thermocapillary valve for droplet production and sorting Physical Review E $\mathbf{7 5} 046302$

[24] Baroud CN, Robert De Saint Vincent M, Delville JP 2007 An optical toolbox for total control of droplet microfluidics Lab on a Chip 8 1029-1033

[25] Garstecki P, Stone HA, Whitesides GM 2005 Mechanism for flow-rate controlled breakup in confined geometries: A route to monodisperse emulsions Physical Review Letters 94164501 $1-4$

[26] Garstecki P, Fuerstman MJ, Stone HA, Whitesides GM 2006 Formation of droplets and bubbles in a microfluidic T-junctionscaling and mechanism of break-up Lab on a Chip 6 437-446 
[27] Guillot P, Colin A 2005 Stability of parallel flows in a microchannel after a T junction Physical Review E $\mathbf{7 2} 066301$ 1-4

[28] Dollet B, Van Hoeve W, Raven JP, Marmottant P, Versluis M 2008 Role of the channel geometry on the bubble pinch-off in flow-focusing devices Physical Review Letters 100034504

[29] De Menech M 2006 Thermocapillary actuation of droplet in a planar microchannel Physical Review E $\mathbf{7 3} 031505$

[30] Fuerstman MJ, Lai A, Thurlow ME, Shevkoplyas SS, Stone HA, Whitesides GM 2007 he pressure drop along rectangular microchannels containing bubbles Lab on a Chip 7 1479-1489

[31] Prakash M, Gershenfeld N 2007 Microfluidic bubble logic Science 315 832-835

[32] Fuerstman MJ, Garstecki P, Whitesides GM 2007 Coding/decoding and reversibility of droplet trains in microfluidic networks Science 315 828-832

[33] Cristobal G, Benoit JP, Joanicot M, Ajdari A 2006 Microfluidic bypass for efficient passive regulation of droplet traffic at a junction Applied Physics Letters 89034104

[34] Jousse F, Farr R, Link DR, Fuerstman MJ, Garstecki P 2006 Bifurcation of droplet flows within capillaries Physical Review E $\mathbf{7 4} 036311$

[35] Schindler M, Ajdari A 2006 Droplet traffic in microfluidic networks: A simple model for understanding and designing Physical Review Letters 100044501

[36] Ting TH, Yap YF, Nguyen NT, Wong TN, Chai JC, Yobas L 2006 Thermally mediated breakup of drops in microchannels Applied Physics Letter 89234101 1-3

[37] Osher S and Sethian JA 1988 Fronts propagating with curvature-dependent speed: Algorithms based on Hamilton-Jacobi formulations Journal of Computational Physics 79 12-49

[38] Yap YF, Chai JC, Wong TN, Toh KC and Zhang HY 2006 A global mass correction scheme for the level-set method Numerical Heat Transfer, Part B: Fundamentals 50 455-472

[39] Brackbill JU, Kothe DB and Zemach C 1992 Flip: A low-dissipation, particle-in-cell method for fluid flow Computer Physics Communications 48 25-38

[40] Nguyen NT, Ting TH, Yap YF, Wong TN, Chai JCK, Ong WL, Zhou JL, Tan SH and Yobas L 2007 Thermally mediated droplet formation in microchannels Applied Physics Letter 91 084102

[41] Patankar SV 1974 Numerical heat transfer and fluid flow Hemisphere, New York 